Supplement of Biogeosciences, 14, 1511-1525, 2017

http://www.biogeosciences.net/14/1511/2017/

doi:10.5194/bg-14-1511-2017-supplement

(C) Author(s) 2017. CC Attribution 3.0 License.

(c) (i)

Supplement of

\title{
Copepod faecal pellet transfer through the meso- and bathypelagic layers in the Southern Ocean in spring
}

\author{
Anna Belcher et al. \\ Correspondence to: Anna Belcher (a.belcher@noc.soton.ac.uk)
}

The copyright of individual parts of the supplement might differ from the CC-BY 3.0 licence. 
Table S1: Absolute number of FPs ( $\mathrm{n}_{\mathrm{FP}}$ ) counted in sediment trap (ST) sample split and Marine Snow Catcher (MSC) samples. Three replicates were counted for ST samples and are presented as mean (standard deviation), where as all FPs collected in the MSC samples were counted. Krill FPs are not included.

\begin{tabular}{cccc}
\hline Cruise & Site & $\begin{array}{c}\text { Sampling } \\
\text { Method }\end{array}$ & nFP \\
\hline JR291 & P2 & MSC & 4 \\
& P2 & MSC & 9 \\
& P2 & MSC & 28 \\
& P3 & MSC & 15 \\
& P3 & MSC & 74 \\
JR304 & P3 & MSC & 120 \\
& P3 & MSC & 252 \\
Dec 2009 & P2 & ST & $422(98)$ \\
& P3 & ST & $1156(195)$ \\
Dec 2010 & P2 & ST & $564(134)$ \\
& P3 & ST & $974(238)$ \\
\hline
\end{tabular}


Table S2: Zooplankton abundance (ind. $\mathrm{m}^{-2}$ ) from Bongo samples (200 $\mathrm{\mu m}$ mesh, 0-200 m) taken in the Scotia Sea, Antarctica during research cruises JR304 and JR291. Species in bold are those used to estimate size distributions of egested faecal pellets.

\begin{tabular}{|c|c|c|c|c|c|c|c|}
\hline \multirow[b]{3}{*}{ Species and Stage } & \multicolumn{5}{|l|}{ JR291 } & \multicolumn{2}{|l|}{ JR304 } \\
\hline & P3 & & & & P2 & & \\
\hline & E072 & E126 & E131 & E137 & E44 & E38 & E40 \\
\hline Rhincalanus gigas male & 27 & 0 & 0 & 0 & 0 & 0 & 0 \\
\hline Rhincalanus gigas female & 712 & 383 & 356 & 329 & 55 & 7 & 465 \\
\hline Rhincalanus gigas $V$ & 219 & 164 & 164 & 246 & 0 & 0 & 383 \\
\hline Rhincalanus gigas IV & 0 & 27 & 0 & 27 & 0 & 0 & 27 \\
\hline Rhincalanus gigas III & 55 & 246 & 274 & 301 & 0 & 0 & 110 \\
\hline Rhincalanus gigas II & 876 & 602 & 274 & 219 & 110 & 0 & 0 \\
\hline Rhincalanus gigas I & 548 & 164 & 137 & 27 & 137 & 0 & 0 \\
\hline Rhincalanus gigas nauplii & 110 & 876 & 1,314 & 438 & 1,971 & 5,256 & 25,405 \\
\hline Calanoides acutus female & 82 & 137 & 164 & 192 & 0 & 301 & 82 \\
\hline Calanoides acutus V & 1,615 & 2,436 & 2,135 & 2,190 & 164 & 0 & 274 \\
\hline Calanoides acutus IV & 3,039 & 1,205 & 1,643 & 1,150 & 575 & 383 & 1,424 \\
\hline Calanoides acutus III & 548 & 219 & 192 & 411 & 602 & 1,341 & 6,351 \\
\hline Calanoides acutus II & 55 & 55 & 164 & 356 & 329 & 3,285 & 10,622 \\
\hline Calanoides acutus I & 0 & 0 & 0 & 274 & 411 & 3,532 & 7,775 \\
\hline Calanus simillimus male & 0 & 0 & 0 & 27 & 0 & 0 & 0 \\
\hline Calanus simillimus female & 27 & 110 & 55 & 27 & 0 & 0 & 137 \\
\hline Calanus simillimus $V$ & 0 & 27 & 27 & 0 & 0 & 0 & 0 \\
\hline Calanus simillimus IV & 0 & 27 & 82 & 82 & 0 & 0 & 0 \\
\hline Calanus simillimus III & 0 & 27 & 0 & 27 & 0 & 0 & 110 \\
\hline Calanus simillimus II & 0 & 27 & 82 & 246 & 0 & 0 & 0 \\
\hline Calanus simillimus I & 0 & 55 & 164 & 274 & 0 & 0 & 0 \\
\hline Calanus propinquus male & 0 & 0 & 0 & 27 & 0 & 0 & 0 \\
\hline Calanus propinquus female & 27 & 0 & 27 & 0 & 0 & 0 & 27 \\
\hline Calanus propinquus $V$ & 110 & 55 & 55 & 27 & 0 & 0 & 0 \\
\hline Calanus propinquus IV & 27 & 55 & 82 & 0 & 27 & 164 & 0 \\
\hline Calanus propinquus III & 0 & 55 & 0 & 0 & 82 & 274 & 438 \\
\hline
\end{tabular}




\begin{tabular}{|c|c|c|c|c|c|c|c|}
\hline \multirow[b]{3}{*}{ Species and Stage } & \multicolumn{5}{|l|}{ JR291 } & \multicolumn{2}{|l|}{ JR304 } \\
\hline & P3 & & & & P2 & & \\
\hline & E072 & E126 & E131 & E137 & $\mathrm{E} 44$ & E38 & E40 \\
\hline Calanus propinquus II & 0 & 0 & 0 & 0 & 27 & 383 & 1,424 \\
\hline Calanus propinquus I & 0 & 0 & 0 & 0 & 0 & 438 & 1,095 \\
\hline Euchaeta antarctica V & 0 & 0 & 0 & 0 & 27 & 0 & 0 \\
\hline Euchaeta antarctica IV & 0 & 0 & 0 & 0 & 27 & 0 & 0 \\
\hline Euchaeta antarctica III & 55 & 55 & 110 & 110 & 0 & 164 & 219 \\
\hline Euchaeta antarctica II & 110 & 164 & 739 & 0 & 0 & 0 & 438 \\
\hline Euchaeta antarctica I & 0 & 82 & 274 & 438 & 0 & 0 & 0 \\
\hline Euchirella rostrata/rostramagna & 0 & 0 & 27 & 0 & 0 & 0 & 0 \\
\hline Haloptilus & 0 & 27 & 0 & 0 & 0 & 82 & 27 \\
\hline Heterorhabdus & 0 & 27 & 27 & 0 & 55 & 0 & 0 \\
\hline Eucalanus longiceps & 0 & 0 & 0 & 0 & 0 & 0 & 27 \\
\hline Metridia gerlachei adult & 164 & 356 & 1,013 & 465 & 55 & 110 & 192 \\
\hline Metridia lucens adult & 0 & 0 & 465 & 246 & 301 & 0 & 0 \\
\hline Metridia male & 110 & 27 & 55 & 0 & 0 & 0 & 0 \\
\hline Metridia I-III & 14,455 & 17,959 & 27,595 & 15,769 & 3,395 & 15,331 & 33,289 \\
\hline Metridia IV-V & 2,847 & 3,066 & 3,504 & 5,256 & 767 & 0 & 1,752 \\
\hline Pleuromamma rob. & 0 & 0 & 55 & 0 & 0 & 0 & 0 \\
\hline Oithona similis & 121,768 & 137,099 & 122,206 & 123,082 & 30,552 & 68,769 & 184,843 \\
\hline Oithona frigida & 876 & 3,504 & 3,066 & 438 & 986 & 2,628 & 0 \\
\hline Oncaea & 10,074 & 9,198 & 16,207 & 4,818 & 14,783 & 4,818 & 12,264 \\
\hline Scolecithricella minor & 903 & 712 & 1,040 & 1,314 & 110 & 137 & 0 \\
\hline Scaphocalanus farrani & 0 & 0 & 27 & 0 & 0 & 0 & 0 \\
\hline Scaphocalanus copepodites & 0 & 0 & 164 & 0 & 0 & 0 & 0 \\
\hline Microcalanus & 1,314 & 2,628 & 8,760 & 2,190 & 876 & 7,884 & 7,008 \\
\hline Ctenocalanus & 55,190 & 33,289 & 21,463 & 25,843 & 5,366 & 17,083 & 65,702 \\
\hline Cteno/Micro copepodites & 0 & 0 & 0 & 0 & 2,628 & 0 & 3,504 \\
\hline Clausocalanus laticeps & 630 & 164 & 438 & 438 & 0 & 110 & 0 \\
\hline Clausocalanus copepodites & 0 & 438 & 438 & 0 & 0 & 0 & 876 \\
\hline Drepanopus 1-3 & 876 & 7,884 & 5,694 & 7,008 & 0 & 0 & 0 \\
\hline Drepanopus 4-6 & 0 & 876 & 438 & 0 & 0 & 0 & 0 \\
\hline
\end{tabular}




\begin{tabular}{lrrrrrrr}
\hline & JR291 & & & & & JR304 \\
P3 & & & & & P2 & \\
Species and Stage & E072 & E126 & E131 & E137 & E44 & E38 & E40 \\
\hline Racovitzanus & 0 & 27 & 0 & 0 & 0 & 0 & 0 \\
Calanus nauplii & 8,322 & 7,446 & 26,719 & 18,835 & 14,126 & 12,264 & 28,909 \\
Cyclopoi nauplii & 0 & 0 & 2,190 & 4,380 & 6,023 & 0 & 0 \\
Thysanoessa calyptopes & 1,205 & 0 & 1,752 & 0 & 329 & 1,424 & 1,697 \\
Thysanoessa spp. & 277 & 137 & 82 & 274 & 110 & 5,858 & 1,451 \\
Euphausia frigida & 86 & 27 & 0 & 0 & 0 & 0 & 0 \\
Euphausia frigida calyptopis & 110 & 0 & 0 & 438 & 0 & 411 & 770 \\
Euphausia frigida furcilia & 55 & 0 & 82 & 192 & 0 & 110 & 55 \\
Euphausia triacantha calyptopis & 0 & 0 & 0 & 0 & 0 & 0 & 27 \\
Chaetognatha & 1,314 & 958 & 1,040 & 931 & 137 & 356 & 1,560 \\
Tomopteris & 0 & 0 & 82 & 438 & 0 & 110 & 0 \\
Pelagobia & 1,752 & 438 & 1,314 & 3,504 & 1,971 & 6,570 & 5,256 \\
Rhynchonerella bongraini & 0 & 0 & 0 & 0 & 0 & 27 & 0 \\
Themisto gaudichaudi & 21 & 0 & 0 & 0 & 0 & 0 & 55 \\
Ostracoda & 14 & 164 & 548 & 356 & 137 & 356 & 301 \\
Pteropoda & 0 & 0 & 0 & 876 & 0 & 1,752 & 876 \\
Limacina (large) & 0 & 0 & 0 & 0 & 0 & 110 & 0 \\
Spongiobranchea & 0 & 27 & 0 & 0 & 0 & 0 & 0 \\
Appendicularians & 6,570 & 10,074 & 20,149 & 35,479 & 2,957 & 15,331 & 33,289 \\
Siphonophora & 0 & 55 & 329 & 110 & 0 & 0 & 0 \\
Fish larvae & 0 & 0 & 0 & 0 & 0 & 27 & 0 \\
Medusae & 0 & 55 & 0 & 0 & 0 & 0 & 0 \\
Primno macropa & 0 & 27 & 0 & 0 & 0 & 0 & 0 \\
\hline
\end{tabular}


Table S3: Sinking velocities and volumes of FPs (excluding krill FPs) collected in Marine Snow Catchers at P2 and P3 during research cruises JR291 and JR304.

\begin{tabular}{|c|c|c|c|c|c|}
\hline Site & $\begin{array}{c}\text { FP volume } \\
\left(\mathrm{mm}^{3}\right)\end{array}$ & $\begin{array}{c}\text { FP sinking } \\
\text { velocity }\left(\mathrm{m} \mathrm{d}^{-1}\right)\end{array}$ & Site & $\begin{array}{c}\text { FP volume } \\
\left(\mathrm{mm}^{3}\right)\end{array}$ & $\begin{array}{c}\text { FP sinking } \\
\text { velocity }\left(\mathrm{m} \mathrm{d}^{-1}\right)\end{array}$ \\
\hline $\mathrm{P} 2$ & 0.040 & 144 & P3 & 0.010 & 75 \\
\hline $\mathrm{P} 2$ & 0.031 & 270 & P3 & 0.027 & 57 \\
\hline $\mathrm{P} 2$ & 0.008 & 52 & P3 & 0.002 & 48 \\
\hline $\mathrm{P} 2$ & 0.040 & 144 & P3 & 0.026 & 87 \\
\hline $\mathrm{P} 2$ & 0.031 & 135 & P3 & 0.002 & 51 \\
\hline $\mathrm{P} 2$ & 0.057 & 134 & P3 & 0.005 & 68 \\
\hline $\mathrm{P} 2$ & 0.019 & 342 & P3 & 0.014 & 49 \\
\hline $\mathrm{P} 2$ & 0.011 & 382 & P3 & 0.028 & 92 \\
\hline $\mathrm{P} 2$ & 0.072 & 247 & P3 & 0.023 & 106 \\
\hline $\mathrm{P} 2$ & 0.044 & 101 & P3 & 0.009 & 24 \\
\hline $\mathrm{P} 2$ & 0.007 & 193 & P3 & 0.091 & 92 \\
\hline $\mathrm{P} 2$ & 0.017 & 116 & P3 & 0.066 & 140 \\
\hline $\mathrm{P} 2$ & 0.035 & 207 & P3 & 0.012 & 57 \\
\hline $\mathrm{P} 2$ & 0.002 & 246 & P3 & 0.006 & 65 \\
\hline $\mathrm{P} 2$ & 0.016 & 61 & P3 & 0.010 & 62 \\
\hline $\mathrm{P} 2$ & 0.001 & 120 & P3 & 0.006 & 64 \\
\hline \multirow[t]{19}{*}{$\mathrm{P} 2$} & 0.003 & 98 & P3 & 0.002 & 47 \\
\hline & & & P3 & 0.037 & 36 \\
\hline & & & P3 & 0.031 & 53 \\
\hline & & & P3 & 0.014 & 122 \\
\hline & & & P3 & 0.021 & 36 \\
\hline & & & P3 & 0.077 & 100 \\
\hline & & & P3 & 0.018 & 62 \\
\hline & & & P3 & 0.026 & 64 \\
\hline & & & P3 & 0.013 & 79 \\
\hline & & & P3 & 0.083 & 227 \\
\hline & & & P3 & 0.286 & 203 \\
\hline & & & P3 & 0.165 & 189 \\
\hline & & & P3 & 0.007 & 100 \\
\hline & & & P3 & 0.006 & 74 \\
\hline & & & P3 & 0.005 & 13 \\
\hline & & & P3 & 0.115 & 106 \\
\hline & & & P3 & 0.021 & 60 \\
\hline & & & P3 & 0.005 & 68 \\
\hline & & & P3 & 0.018 & 79 \\
\hline
\end{tabular}




\begin{tabular}{llc} 
P3 & 0.006 & 49 \\
P3 & 0.009 & 64 \\
P3 & 0.003 & 155 \\
P3 & 0.005 & 222 \\
P3 & 0.256 & 144 \\
P3 & 0.002 & 82 \\
P3 & 0.006 & 133 \\
\hline
\end{tabular}




\section{Supplementary Figures}
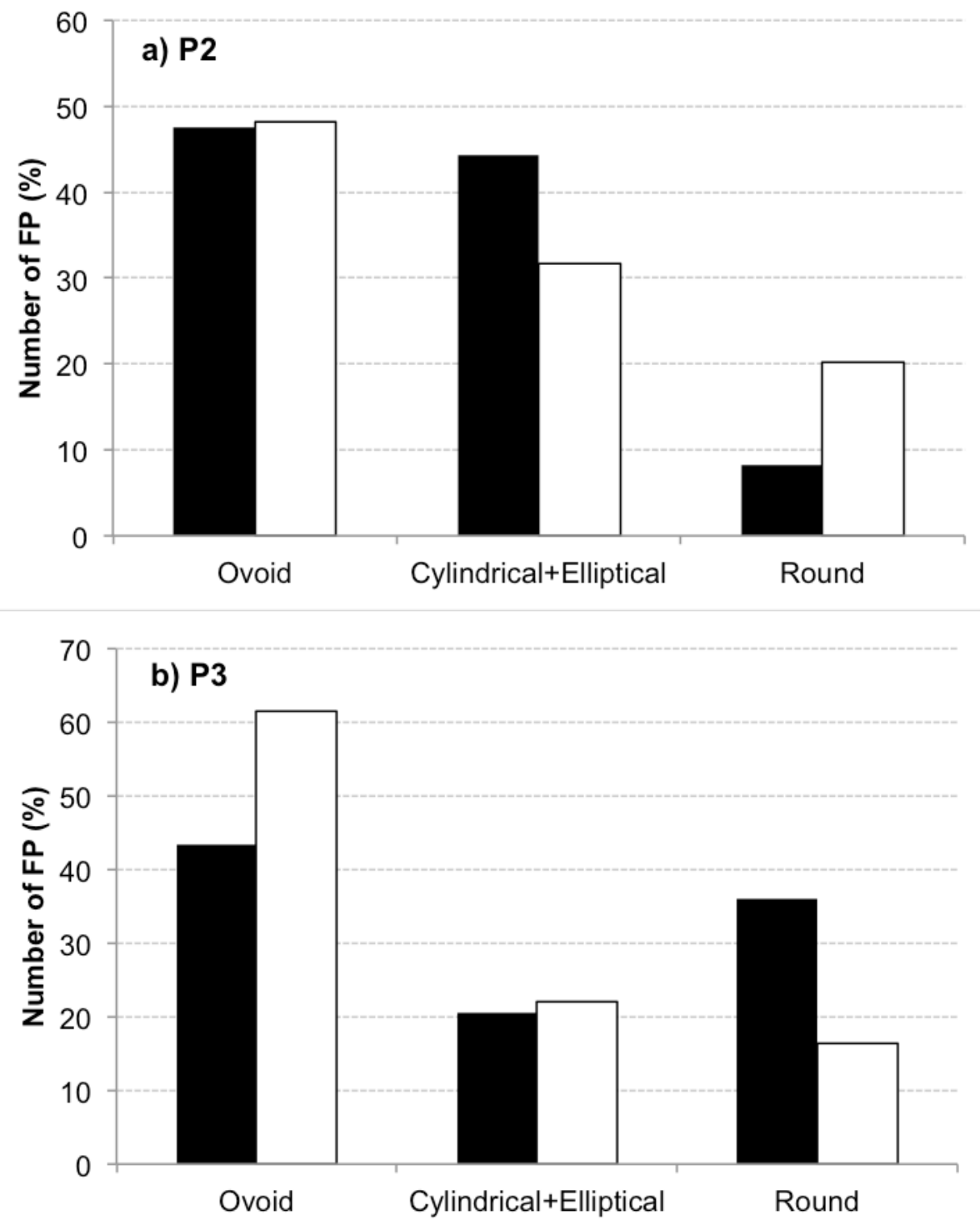

Figure S1: Comparison of sediment trap faecal pellet (FP) morphologies measured in this study (2013 and 2014, black) with those measured historically (2009 and 2010, white) at a) P2 and b) P3. Both studies are means of November and December data. The percent (\%) of FPs in each category is broadly consistent between study years (paired t-test p>0.5) providing support for our use of historical data for size comparisons with Marine Snow Catcher data collected in 2013 and 2014. 
a) $\mathrm{P} 2$

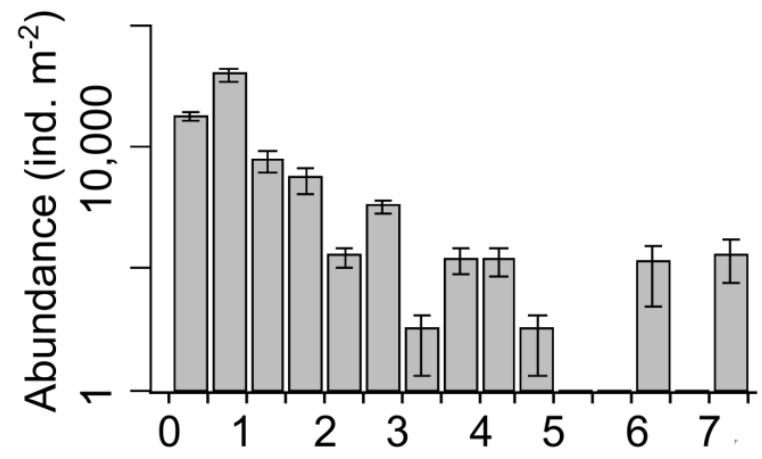

b) $\mathrm{P} 3$

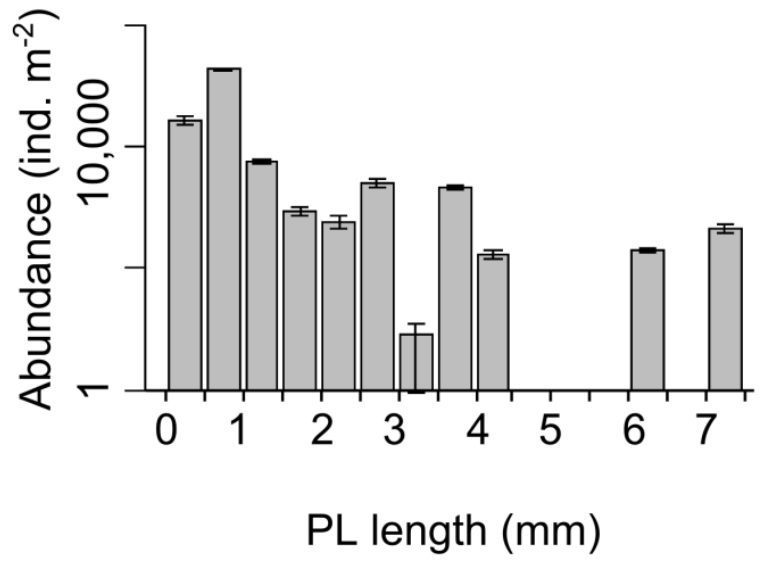

Figure S2: Mesozooplankton abundances in the Scotia Sea. Average $( \pm \mathrm{SE})$ abundance (ind. $\left.\mathrm{m}^{-2}\right)$ from Bongo net tows $(0-200 \mathrm{~m}$, $200 \mathrm{\mu m}$ mesh) taken during cruises JR291 and JR304 for a) P2 and b) P3. Note the log scale on the $y$ axis. 